\title{
SMOOTHNESS OF THE BILLIARD BALL MAP FOR STRICTLY CONVEX DOMAINS NEAR THE BOUNDARY
}

\author{
VALERY KOVACHEV
}

(Communicated by Kenneth R. Meyer)

\begin{abstract}
The billiard ball map in bounded strictly convex domains in $\mathbf{R}^{n}$ with boundaries of class $C^{k}, k \geq 2$, is considered and its smoothness of class $C^{k-1}$ up to the boundary is proved.
\end{abstract}

1. Introduction. It is well known that the billiard ball map for plane billiards in domains bounded by strictly convex smooth curves with everywhere nonzero curvature is smooth up to the boundary (see [1]). More precisely, if the boundary is of class $C^{k}, k \geq 2$, then the billiard ball map is of class $C^{k-1}$ (see [4]). This property of such billiards is systematically used in the study of their ergodic properties [2, 3, 4].

In the present paper we obtain an analogous result for $n$-dimensional domains. It guarantees the applicability of the theory elaborated in [5] (see [6]) to billiards in strictly convex bounded regions in $\mathbf{R}^{n}$ with boundary of class $C^{k}, k \geq 3$, in particular Pesin's entropy formula $[\mathbf{8}, \mathbf{9}, \mathbf{7}]$ holds for such billiards.

2. Main result. Assume that $\Omega$ is a strictly convex bounded domain in $\mathbf{R}^{n}$ with boundary $\partial \Omega$ of class $C^{k}, k \geq 2$. Denote by $n_{z}$ the unit inward normal to $\partial \Omega$ at the point $z \in \Omega$ and define the set

$$
\Sigma=\left\{(z, e) \in \partial \Omega \times S^{n-1} ;\left\langle e, n_{z}\right\rangle_{n} \geq 0\right\}
$$

where $\langle\cdot, \cdot\rangle_{n}$ is the scalar product in $\mathbf{R}^{n}$. Then $\Sigma$ is a compact $(2 n-2)$-dimensional manifold of class $C^{k}$ with boundary $\partial \Sigma$ of class $C^{k-1}$ which is defined by the equation $\left\langle e, n_{z}\right\rangle_{n}=0$.

Recall now the definition of the billiard ball map $B: \Sigma \rightarrow \Sigma$ (see [4]). From the convexity of $\Omega$ it follows that for each $(z, e) \in \Sigma \backslash \partial \Sigma$ there exists a unique $t>0$ such that the point

$$
z^{*}=z+t e
$$

belongs to $\partial \Omega$. For $(z, e) \in \partial \Sigma$ we assume $t=0$ and $z^{*}=z$. Then define the map $B: \Sigma \rightarrow \Sigma,(z, e) \rightarrow\left(z^{*}, e^{*}\right)$, where $z^{*}$ was defined above and $e^{*}=s_{z^{*}}(e)$ where $s_{z^{*}}$ is the symmetry with respect to the hyperplane $T_{z^{*}} \partial \Omega$. Obviously $B$ is the identity on $\partial \Sigma$. Moreover, it is well known that $B$ is a diffeomorphism of $\Sigma \backslash \partial \Sigma$ which is continuous up to $\partial \Sigma$. Further on we prove the following theorem.

Received by the editors December 29, 1986 and, in revised form, April 10, 1987.

1980 Mathematics Subjcct Classification (1985 Revision). Primary 34C35, 58C25; Secondary $58 \mathrm{~F} 17$.

Key words and phrases. Smoothness near the boundary, billiard, convex. 
THEOREM 1. Let $\Omega$ be a strictly convex bounded domain in $\mathbf{R}^{n}$ with boundary of class $C^{k}, k \geq 2$. Then the billiard ball map $B$ is a diffeomorphism of class $C^{k-1}$ of the compact manifold $\Sigma$ onto itself.

3. Proof of Theorem 1. Take an arbitrary point $M \in \partial \Omega$. From the strict convexity of $\Omega$ it follows that there exists a neighbourhood $U_{M}$ of $M$ such that $\partial \Omega \cap U_{M}$ can be represented by the equation

$$
y=g(x)
$$

where $g(x)$ is a function of class $C^{k}$ defined in an $(n-1)$-dimensional neighbourhood of the origin such that $g(0)=0, \partial g(0)=0$ and the matrix $\partial^{2} g(0)$ is positively definite, i.e. there exists a constant $C>0$ such that $\left\langle\partial^{2} g(0) \xi, \xi\right\rangle \geq 2 C|\xi|^{2}$ for all $\xi \in$ $\mathbf{R}^{n-1}$. Here $\langle\cdot, \cdot\rangle$ is the scalar product in $\mathbf{R}^{n-1}$. Choose a convex neighbourhood $V$ of 0 in $\mathbf{R}^{n-1}$ such that for all $x \in V$ we have $z=(x, g(x)) \in \partial \Omega \cap U_{M}$,

$$
|\partial g(x)|<\min \left((15 / n)^{1 / 2} / 4,15^{-1 / 2}\right)
$$

and

$$
\left\langle\partial^{2} g(x) \xi, \xi\right\rangle \geq C|\xi|^{2}
$$

for all $\xi \in \mathbf{R}^{n-1}$. Let $V^{\prime}$ be a neighbourhood of 0 in $\mathbf{R}^{n-1}$ such that $\overline{V^{\prime}} \subset V$ and denote $U_{M}^{\prime}=\left\{(x, g(x)) ; x \in V^{\prime}\right\}$. From the covering $\left\{U_{M}^{\prime}\right\}_{M \in \partial \Omega}$ of $\partial \Omega$ we can choose a finite subcovering and further on all considerations will be in one of these neighbourhoods denoted by $U^{\prime}$ such that $\overline{U^{\prime}} \subset U=\{(x, g(x)) ; x \in V\}$.

The unit inward normal at the point $z=(x, g(x)) \in U$ is given by

$$
\left.n_{z}=(-\partial g(x), 1) /(1+|\partial g(x)|)^{2}\right)^{1 / 2} \text {. }
$$

For any vector $e \in S^{n-1}$ such that $(z, e) \in \Sigma$ put $e=(\xi, \eta)=\left(\xi_{1}, \ldots, \xi_{n-1}, \eta\right) \in$ $\mathbf{R}_{\xi}^{n-1} \times \mathbf{R}_{\eta}$ and denote

$$
\varepsilon=\left\langle e, n_{z}\right\rangle_{n} .
$$

Here $\varepsilon$ is a nonnegative number vanishing only on $\partial \Sigma$. From (5) and (6) we obtain

$$
\eta=\varepsilon\left(1+|\partial g(x)|^{2}\right)^{1 / 2}+\langle\partial g(x), \xi\rangle .
$$

Since we investigate the billiard ball map near $\partial \Sigma$, we can assume $0 \leq \varepsilon<1 / 4$. Then from (7) and (3) it follows that

$$
\begin{aligned}
|\eta| & \leq \varepsilon\left(1+|\partial g(x)|^{2}\right)^{1 / 2}+|\partial g(x)||\xi| \leq(1+1 / 15)^{1 / 2} / 4+15^{-1 / 2}|\xi| \\
& =15^{-1 / 2}(1+|\xi|) .
\end{aligned}
$$

Since $e=(\xi, \eta) \in S^{n-1}$, then $1=|\xi|^{2}+|\eta|^{2} \leq|\xi|^{2}+(1+|\xi|)^{2} / 15$ which implies $8|\xi|^{2}+|\xi|-7 \geq 0$ or $|\xi| \geq 7 / 8$.

Denote

$$
\begin{aligned}
& W_{j}=\left\{(x, g(x), \xi, \eta) \in U \times S^{n-1} ; x \in V,\left|\xi_{j}\right|>3 n^{-1 / 2} / 4\right. \\
& \left.\eta=\varepsilon\left(1+|\partial g(x)|^{2}\right)^{1 / 2}+\langle\partial g(x), \xi\rangle, 0 \leq \varepsilon<1 / 4\right\}, \quad j=1,2, \ldots, n-1, \\
& W_{j}^{\prime}=\left\{(x, g(x), \xi, \eta) \in U^{\prime} \times S^{n-1} ; x \in V^{\prime},\left|\xi_{j}\right|>7 n^{-1 / 2} / 8,\right. \\
& \left.\eta=\varepsilon\left(1+|\partial g(x)|^{2}\right)^{1 / 2}+\langle\partial g(x), \xi\rangle, 0 \leq \varepsilon<\varepsilon_{0}\right\}, \quad j=1,2, \ldots, n-1,
\end{aligned}
$$


where the constant $\varepsilon_{0}<1 / 4$ will be determined below. Obviously

$$
\begin{aligned}
& U \times\left\{e \in S^{n-1} ; 0 \leq\left\langle e, n_{z}\right\rangle_{n}<1 / 4\right\}=\bigcup_{j=1}^{n-1} W_{j}, \\
& U^{\prime} \times\left\{e \in S^{n-1} ; 0 \leq\left\langle e, n_{z}\right\rangle_{n}<\varepsilon_{0}\right\}=\bigcup_{j=1}^{n-1} W_{j}^{\prime} .
\end{aligned}
$$

Thus the union of all $W_{j}^{\prime}$ (or $W_{j}$ ) related to a finite covering $\left\{U^{\prime}\right\}$ of $\partial \Omega$ (or to the respective convex neighbourhoods $U \supset \overline{U^{\prime}}$ ) provides a finite covering of a neighbourhood of $\partial \Sigma$ in $\Sigma$.

Below we fix an index $j: 1 \leq j \leq n-1$, and denote

$$
\phi(x, \varepsilon, \xi)=|\xi|^{2}+\left(\varepsilon\left(1+|\partial g(x)|^{2}\right)^{1 / 2}+\langle\partial g(x), \xi\rangle\right)^{2}-1 .
$$

Then the partial derivative $\phi_{\xi_{j}}(x, \varepsilon, \xi)$ equals

$$
2 \xi_{j}+2\left(\varepsilon\left(1+|\partial g(x)|^{2}\right)^{1 / 2}+\langle\partial g(x), \xi\rangle\right) g_{x_{j}}(x)
$$

and for $(z, e) \in W_{j}$ we have

$$
\begin{aligned}
\left|\phi_{\xi_{j}}(x, \varepsilon, \xi) / 2\right| & \geq\left|\xi_{j}\right|-\left|\varepsilon\left(1+|\partial g(x)|^{2}\right)^{1 / 2}+\langle\partial g(x), \xi\rangle \| g_{x_{j}}(x)\right| \\
& \geq 3 n^{-1 / 2} / 4-15^{-1 / 2}(1+|\xi|)(15 / n)^{1 / 2} / 4 \\
& \geq 3 n^{-1 / 2} / 4-2 n^{-1 / 2} / 4=n^{-1 / 2} / 4>0 .
\end{aligned}
$$

By the implicit function theorem there exists $\xi_{j}\left(x, \xi_{1}, \ldots, \xi_{j-1}, \xi_{j+1}, \ldots, \xi_{n-1}, \varepsilon\right)$ a function of class $C^{k-1}$ satisfying $\phi(x, \varepsilon, \xi)=0$. Thus in $W_{j}$ and $W_{j}^{\prime}$ we can use the local coordinates $\left(x, \xi_{1}, \ldots, \xi_{j-1}, \xi_{j+1}, \ldots, \xi_{n-1}, \varepsilon\right)$ and in any of these neighbourhoods the boundary $\partial \Sigma$ is given by $\varepsilon=0$.

From $B(z, e)=(z, e)$ on $\partial \Sigma$ where $\varepsilon=0$, the continuity of $B$ up to the boundary and the fact that $\overline{U^{\prime}} \subset U$ it follows that there exists a positive number $\varepsilon_{0}<1 / 4$ such that for all $z \in U^{\prime}, e \in S^{n-1}: 0<\left\langle e, n_{z}\right\rangle_{n}<\varepsilon_{0}$ we have $z^{*} \in U$. Here $z^{*}$ is the point determined by $(1)$.

Now assume that $(z, e) \in W_{j}^{\prime}$. In the local coordinates chosen above (1) can be written as

$$
\begin{aligned}
x^{*} & =x+t \xi, \\
g\left(x^{*}\right) & =g(x)+t \eta .
\end{aligned}
$$

In view of (7) last equality becomes

$$
g(x+t \xi)=g(x)+t\left(\varepsilon\left(1+|\partial g(x)|^{2}\right)^{1 / 2}+\langle\partial g(x), \xi\rangle\right) .
$$

By Taylor's formula we have

$$
g(x+t \xi)=g(x)+t \int_{0}^{1}\langle\partial g(x+s t \xi), \xi\rangle d s .
$$

Thus from (11) we obtain

$$
\int_{0}^{1}\langle\partial g(x+s t \xi), \xi\rangle d s=\varepsilon\left(1+|\partial g(x)|^{2}\right)^{1 / 2}+\langle\partial g(x), \xi\rangle .
$$


Since the derivative of the left-hand side with respect to $t$ is

$$
\int_{0}^{1} s\left\langle\partial^{2} g(x+s t \xi) \xi, \xi\right\rangle d s \geq \int_{0}^{1} s C|\xi|^{2} d s \geq C\left(\frac{7}{8}\right)^{2} / 2=\frac{49 C}{128}>0
$$

from the implicit function theorem it follows that $t$ is a function of $(x, \xi, \varepsilon)$ of class $C^{k-1}$, hence by (9) $x^{*}$ is a function of $(x, \xi, \varepsilon)$ of class $C^{k-1}$ for $0 \leq \varepsilon<\varepsilon_{0}$. In order to express more explicitly the dependence of $t$ and $x^{*}$ on $\varepsilon$, replace (12) by the expansion

$$
g(x+t \xi)=g(x)+t\langle\partial g(x), \xi\rangle+t^{2} \int_{0}^{1}(1-s)\left\langle\partial^{2} g(x+s t \xi) \xi, \xi\right\rangle d s
$$

Hence $t$ satisfies the equation

$$
t \int_{0}^{1}(1-s)\left\langle\partial^{2} g(x+s t \xi) \xi, \xi\right\rangle d s=\varepsilon\left(1+|\partial g(x)|^{2}\right)^{1 / 2}
$$

This shows that $t=O(\varepsilon), x^{*}=x+O(\varepsilon)$ uniformly on $(z, e) \in W_{j}^{\prime}$.

Now we shall express

$$
\begin{aligned}
\varepsilon^{*} & =-\left\langle n_{z^{*}}, e\right\rangle_{n} \\
& =\left(\left\langle\partial g\left(x^{*}\right), \xi\right\rangle-\varepsilon\left(1+|\partial g(x)|^{2}\right)^{1 / 2}-\langle\partial g(x), \xi\rangle\right) /\left(1+\left|\partial g\left(x^{*}\right)\right|^{2}\right)^{1 / 2} .
\end{aligned}
$$

This representation allows us to conclude that $\varepsilon^{*}$ is a function of $(x, \xi, \varepsilon)$ of class $C^{k-1}$. By Taylor's formula

$$
\partial g\left(x^{*}\right)=\partial g(x+t \xi)=\partial g(x)+t \int_{0}^{1} \partial^{2} g(x+s t \xi) \xi d s
$$

and by (14) we obtain

$$
\varepsilon^{*}=\varepsilon\left(\frac{1+|\partial g(x)|^{2}}{1+\left|\partial g\left(x^{*}\right)\right|^{2}}\right)^{1 / 2} \frac{\int_{0}^{1} s\left\langle\partial^{2} g(x+s t \xi) \xi, \xi\right\rangle d s}{\int_{0}^{1}(1-s)\left\langle\partial^{2} g(x+s t \xi) \xi, \xi\right\rangle d s}
$$

hence $\varepsilon^{*}=\varepsilon+O\left(\varepsilon^{2}\right)$ uniformly on $(z, e) \in W_{j}^{\prime}$. This implies that for $\varepsilon_{0}$ small enough we shall have $\varepsilon^{*}<1 / 4$ for any $(z, e) \in W_{j}^{\prime}$.

Further on, we have $e^{*}=e-2 \varepsilon^{*} n_{z^{*}}$, hence $\xi^{*}$ is a function of $(x, \xi, \varepsilon)$ of class $C^{k-1}$. Write the equality of the first $n-1$ coordinates of $e^{*}$ :

$$
\xi^{*}=\xi+2 \varepsilon \frac{\left(1+|\partial g(x)|^{2}\right)^{1 / 2}}{1+\left|\partial g\left(x^{*}\right)\right|^{2}} \frac{\int_{0}^{1} s\left\langle\partial^{2} g(x+s t \xi) \xi, \xi\right\rangle d s}{\int_{0}^{1}(1-s)\left\langle\partial^{2} g(x+s t \xi) \xi, \xi\right\rangle d s} \partial g\left(x^{*}\right) .
$$

Last equality shows that $\xi^{*}=\xi+O(\varepsilon)$ uniformly on $(z, e) \in W_{j}^{\prime}$. This implies that for $\varepsilon_{0}$ small enough we shall have $\left|\xi_{j}^{*}\right|>3 n^{-1 / 2} / 4$, hence $\left(z^{*}, e^{*}\right) \in W_{j}$.

Thus we have shown that $B$ is a map of class $C^{k-1}$ from $W_{j}^{\prime}$ into $W_{j}$. Since $W_{j}^{\prime}$ is a sufficiently small neighbourhood of an arbitrary point of $\partial \Sigma$, this proves the smoothness of $B$ near the boundary. 


\section{REFERENCES}

1. V. F. Lazutkin, Convex billiards and eigenfunctions of the Laplace operator, Ed. Leningrad University, 1981. (Russian)

2. Izv. 7 (1973), 185-215.

3. M. M. Dvorin and V. F. Lazutkin, The existence of an infinite number of elliptic and hyperbolic periodic trajectories for a convex billiard, Funct. Anal. Appl. 7 (1973), 103-109.

4. R. Douady, Application du théorème des tores invariants, Thèse de 3ème cycle, Université Paris VII, 1982.

5. A. Katok and J.-M. Strelcyn, Smooth maps with singularities: Invariant manifolds, entropy and billiards, Lecture Notes in Math., vol. 1222, Springer-Verlag, Berlin and New York, 1986.

6. J.-M. Strelcyn, Les applications différentiables avec singularités; les sous-variétés invariantes, l'entropie et les billiards, Travaux en Cours, Hermann, Paris, 1985, pp. 93-111.

7. F. Ledrappier and J.-M. Strelcyn, A proof of the estimation from below in Pesin's entropy formula, Ergodic Theory Dynamical Systems 2 (1982), 203-219.

8. Y. Pesin, Characteristic Lyapunov exponents and smooth ergodic theory, Russian Math. Surveys 32 (1977), 55-114.

9. R. Mañe, A proof of Pesin's formula, Ergodic Theory Dynamical Systems 1 (1981), 95-102.

Institute of Mathematics, Bulgarian ACAdemy of Sciences, P.O. Box 373, 1090 SOFIA, BULGARIA 